\title{
Sampling and Analytical Method for Alpha-Dicarbonyl Flavoring Compounds via Derivatization with o-Phenylenediamine and Analysis Using GC-NPD
}

\author{
Stephanie M. Pendergrass ${ }^{1}$ and Jeffrey A. Cooper $^{2}$ \\ ${ }^{1}$ National Institute for Occupational Safety and Health Centers for Disease Control and Prevention, Cincinnati, OH 45226, USA \\ ${ }^{2}$ Bureau Veritas North America, Novi, MI 48375, USA \\ Correspondence should be addressed to Stephanie M. Pendergrass; smp5@cdc.gov
}

Received 3 December 2015; Revised 25 February 2016; Accepted 4 April 2016

Academic Editor: Lidwien A. M. Smit

Copyright (C) 2016 S. M. Pendergrass and J. A. Cooper. This is an open access article distributed under the Creative Commons Attribution License, which permits unrestricted use, distribution, and reproduction in any medium, provided the original work is properly cited.

\begin{abstract}
A novel methodology is described for the sampling and analysis of diacetyl, 2,3-pentanedione, 2,3-hexanedione, and 2,3heptanedione. These analytes were collected on $o$-phenylenediamine-treated silica gel tubes and quantitatively recovered as the corresponding quinoxaline derivatives. After derivatization, the sorbent was desorbed in $3 \mathrm{~mL}$ of ethanol solvent and analyzed using gas chromatography/nitrogen-phosphorous detection (GC/NPD). The limits of detection (LOD) achieved for each analyte were determined to be in the range of 5-10 nanograms/sample. Evaluation of the on-tube derivatization procedure indicated that it is unaffected by humidities ranging from $20 \%$ to $80 \%$ and that the derivatization procedure was quantitative for analyte concentrations ranging from $0.1 \mu \mathrm{g}$ to approximately $500 \mu \mathrm{g}$ per sample. Storage stability studies indicated that the derivatives were stable for 30 days when stored at both ambient and refrigerated temperatures. Additional studies showed that the quinoxaline derivatives were quantitatively recovered when sampling up to a total volume of $72 \mathrm{~L}$ at a sampling rate of $50 \mathrm{cc} / \mathrm{min}$. This method will be important to evaluate and monitor worker exposures in the food and flavoring industry. Samples can be collected over an 8-hour shift with up to $288 \mathrm{~L}$ total volume collected regardless of time, sampling rate, and/or the effects of humidity.
\end{abstract}

\section{Introduction}

In August 2000, the National Institute for Occupational Safety and Health (NIOSH) received a request for technical assistance (HETA \# 00-0401) in an investigation of severe obstructive lung disease (bronchiolitis obliterans) in former workers of a microwave popcorn plant in Missouri [1]. NIOSH was asked to investigate a cluster of past and present employees experiencing severe respiratory symptoms after working in microwave popcorn processing facilities over a period of 3 months to 3 years [2]. A NIOSH medical and environmental survey at the plant in November 2000 demonstrated a strong exposure-response relationship between quantities of estimated cumulative exposure to diacetyl (a volatile butter flavoring chemical contaminating the air in the plant) and the frequency of airway obstruction on spirometry tests [1].

NIOSH method 2557, an air sampling method that uses Anasorb Carbon Molecular Sieve (CMS) sorbent tubes, was developed based on an urgent need for a method to collect and quantitate exposures and evaluate subsequent engineering control effectiveness [3]. This method was used extensively in the field for a number of years. Subsequent field evaluation work suggested a tendency of NIOSH method 2557 to underestimate the true concentration of diacetyl in air [4]. Additional laboratory studies identified that this method had reduced recoveries when samples were collected in moderate-to-high humidity environments. A NIOSH laboratory-based study and a chamber study with generated atmospheres established a correction method for previously 
collected data with the initial NIOSH method [5]. Concurrently, the Occupational Safety and Health Administration (OSHA) developed method PV2118 that collected diacetyl on a silica gel sorbent. While the method exhibited good storage stability for diacetyl, it had limitations in sampling time/volume because of the collection of water during air sampling [6].

In an effort to address the humidity concerns encountered by the NIOSH and OSHA methods, OSHA developed another method for the collection and analysis of diacetyl on specially dried silica gel tubes (2 tubes in series) [7]. By using the dried silica gel tubes in series, this OSHA method addressed migration issues encountered when a single silica gel tube was used. All of these methods utilize gas chromatography equipped with flame ionization detection (GC/FID) for sample analyses.

In 2011, NIOSH published a draft criteria document titled "Criteria for a Recommended Standard: Exposure to Diacetyl and 2,3-Pentanedione" that contained a draft NIOSH Recommended Exposure Limit (REL) of $5 \mathrm{pbb}$, $8 \mathrm{hr}$-TWA for diacetyl [8]. The criteria document recommended OSHA method 1012 for sampling diacetyl exposures. This method utilizes $o$-(2,3,4,5,6-pentafluorobenzyl) hydroxylamine hydrochloride (PFBHA) to derivatize diacetyl followed by analysis using gas chromatography with electron capture detection (GC-ECD) [9]. OSHA method 1012 has limitations in sampling time and capacity due to the potential collection of water during air sampling, as well as an extended derivatization time up to 36 hours.

To address the limitations in diacetyl sampling, a research protocol was designed based upon the derivatization of diacetyl (which was subsequently applied to analogous alphadicarbonyl compounds) with $o$-phenylenediamine (o-PDA). Several research groups have documented the conversion of alpha-dicarbonyl compounds into the corresponding quinoxalines using $o$-PDA [10-12].

Therefore, the focus of this research project was to develop a method for the collection, derivatization, and stabilization of diacetyl and the other alpha-dicarbonyl compounds (2,3-pentanedione, 2,3-hexanedione, and 2,3heptanedione) as quinoxaline derivatives.

\section{Methods}

2.1. Apparatus. Gas chromatographic (GC) analyses were conducted using a Hewlett Packard Model 5890 Series II GC with a nitrogen/phosphorus detector (NPD) (Agilent Tech., Avondale, PA) equipped with a $30 \mathrm{~m}$ RTX-5 fused silica capillary column $(0.25 \mathrm{~mm}$ ID, $1 \mu \mathrm{m}$ film) (Restek Corp., Bellefonte, PA).

Baseline separation and optimal resolution of diacetyl, 2,3-pentanedione, 2,3-hexanedione, and 2,3-heptanedione from the excess derivatizing reagent were achieved using the following parameters. The $\mathrm{GC}$ oven temperature program was ramped up from $50^{\circ} \mathrm{C}$ (held $\left.1 \mathrm{~min}\right)$ to $200^{\circ} \mathrm{C}\left(10^{\circ} \mathrm{C} / \mathrm{min}\right.$ ) and held for $2 \mathrm{~min}$. The injection port temperature was set at $240^{\circ} \mathrm{C}$, the detector temperature at $300^{\circ} \mathrm{C}$, and the carrier gas (helium) to a flow rate of $1.36 \mathrm{~mL} / \mathrm{min}$. The injection solvent was ethanol, which was also used as the method desorption solvent. A splitless GC injection port liner was used and $1 \mu \mathrm{L}$ aliquot was injected.

2.2. Reagents. Diacetyl (97\%, CAS \# 431-03-8), o-PDA (99.5\%, CAS \# 95-54-5), 2,3-dimethylquinoxaline (97\%, CAS \# 2379-55-7), 2,3-pentanedione (97\%, CAS \# 600-14-6), 2,3hexanedione ( $\geq 93 \%$, CAS \# 3848-24-6), 2,3-heptanedione ( $\geq 97 \%$, CAS \# 96-04-8), and ethanol (99.5\%, CAS \# 6417-5) were purchased from Sigma-Aldrich Chemical Co. (Milwaukee, WI).

Commercially available silica gel sorbent tubes (SKC \# 226-183) and specially prepared $o$-PDA-treated silica gel sorbent tubes (SKC \# CPM021109-001) were obtained from SKC, Inc. (Eighty Four, PA). The commercially available silica gel sorbent tubes contain two sections of silica gel (600 mg front section and $600 \mathrm{mg}$ back section). The $o^{-}$ PDA- (nominally $0.1 \%$ by weight) treated silica gel sorbent tubes contain two sections of treated silica gel $(520 \mathrm{mg}$ front section, a PUF separator, and $260 \mathrm{mg}$ back section). Ethanol (99.5\%, CAS \# 64-17-5) was used as the solvent for all spiking solutions and as the eluting solvent. For all sorbent tubes, the front section (A) and back section (B) were desorbed separately in $3 \mathrm{~mL}$ of ethanol in autosampler vials (sealed) and placed on a shaker for 90 minutes to facilitate desorption. Analyte spikes, depending on the study, were placed on the front section of the sorbent tube, or onto the initial glass wool plug, or from generated aerosols. For each concentration level evaluated, six samples $(N=6)$ were prepared. A Teflon magnetic stir bar $(12.7 \mathrm{~mm} \times 7.9 \mathrm{~mm}, \mathrm{VWR}$, Inc. $)$ was placed in each vial. After the desorption period, a portion $(1 \mathrm{~mL})$ of each sample was transferred to $2 \mathrm{~mL}$ autosampler vials for analysis using GC-NPD ( $1 \mu \mathrm{L}$ injection).

2.3. Procedures. In order to address the identified limitations of current methods, a number of laboratory evaluations were conducted: (a) determination of LOD and Limit of Quantitation (LOQ), (b) determination of the efficacy of the postsampling derivatization of diacetyl collected on large untreated silica gel tubes, (c) determination of diacetyl, 2,3pentanedione, and 2,3-hexanedione recovery from $o$-PDAcoated silica gel sorbent, (d) determination of the effects of high humidity on the derivatization process, (e) determination of the maximum collection capacity of the coated silica gel sorbent, and (f) determination of analyte storage stability.

2.3.1. LOD/LOQ Determination. Using GC-NPD, eight standards (analyzed in duplicate) were prepared and derivatized on-tube ranging from $2.65 \mathrm{ng} / \mathrm{mL}$ to $662.5 \mathrm{ng} / \mathrm{mL}$ for diacetyl, from $10 \mathrm{ng} / \mathrm{mL}$ to $100.7 \mathrm{ng} / \mathrm{mL}$ for 2,3 -pentanedione, and from $5 \mathrm{ng} / \mathrm{mL}$ to $201.6 \mathrm{ng} / \mathrm{mL}$ for 2,3-hexanedione.

For LOD and LOQ determination, analytical standards were prepared by serial dilution for diacetyl, 2-pentanedione, and 2,3-hexanedione solutions and $1 \mu \mathrm{L}$ aliquots were spiked directly onto the sorbent tube. After equilibration, the sorbent sections were desorbed in $2 \mathrm{~mL}$ of ethanol for 60 minutes [13].

2.3.2. Recovery Study (Untreated Silica Gel Sorbent Tubes). Initially, untreated silica gel sorbent tubes were prepared for 
TABLE 1: Preparation of analyte stock solutions and spiking volumes for recovery studies.

\begin{tabular}{lccc}
\hline Analyte & $\begin{array}{c}\text { Spiking level } \\
(\mu \mathrm{g})\end{array}$ & $\begin{array}{c}\text { Sample } \\
\text { volume } \\
\text { spiked }(\mu \mathrm{L})\end{array}$ & $\begin{array}{c}\text { Amount of } \\
\text { analyte }(\mu \mathrm{g})\end{array}$ \\
\hline \multirow{2}{*}{ Diacetyl } & 0.1 & 0.5 & 0.0955 \\
& 10 & 50 & 9.55 \\
& 100 & 10 & 95.5 \\
& 250 & 25 & 239 \\
2,3- & 500 & 50 & 478 \\
Pentanedione & 0.1 & 0.5 & 0.0959 \\
& 10 & 50 & 9.59 \\
& 100 & 10 & 95.9 \\
& 250 & 25 & 240 \\
2,3- & 500 & 50 & 480 \\
Hexanedione & 0.1 & 0.5 & 0.117 \\
& 10 & 50 & 11.7 \\
& 100 & 10 & 117 \\
& 250 & 25 & 292 \\
& 500 & 50 & 584 \\
\hline
\end{tabular}

desorption efficiency (DE) studies after the determination of the method LOD/LOQ using GC-NPD. Spikes were prepared at the following levels: $0.0955 \mu \mathrm{g}, 9.55 \mu \mathrm{g}, 95.5 \mu \mathrm{g}, 239 \mu \mathrm{g}$, and $478 \mu \mathrm{g}$.

2.3.3. Recovery Study (o-PDA-Treated Silica Gel Sorbent Tubes). For the initial desorption efficiency study, where the custom-made and $o$-PDA-treated silica gel tubes (SKC \# CPM021109-001) containing 0.1\% o-PDA by weight were used, the desorption efficiencies for diacetyl, 2,3pentanedione, 2,3-hexanedione, and 2,3-heptanedione were evaluated. Spikes were prepared ranging from approximately $0.1 \mu \mathrm{g}$ to $500 \mu \mathrm{g}$ (10 to $100 \mu \mathrm{g}$ for 2,3-heptanedione) and are listed in Table 1 . The ensuing sample preparation and analyses were the same as described in the previous section.

2.3.4. Low-Level Recovery Studies. To further define the lower sample recovery limits, a low-level recovery study (0.1 to $1 \mu \mathrm{g})$ was conducted for each analyte. Using the custommade, unwashed, and dried $o$-PDA-treated silica gel tubes (SKC \# CPM021109-001), diacetyl, 2,3-pentanedione, 2,3hexanedione, and 2,3-heptanedione were evaluated.

2.3.5. Studies of the Effect of Humidity on Recovery. To evaluate the effects of relative humidity on sample collection and recovery, the treated sorbent tubes were placed on an air sampling manifold (Miller-Nelson Flow Temperature Humidity Control System, Model HCS-401) and the flow rate of the manifold was adjusted to $50 \mathrm{cc} / \mathrm{min}$. Each tube was spiked with a solution containing diacetyl, 2,3-pentanedione, 2,3hexanedione, and 2,3-heptanedione at multiple levels ranging from $0.1 \mu \mathrm{g}$ to $500 \mu \mathrm{g}(100 \mu \mathrm{g}$ for 2,3-heptanedione since it was a minor component in all samples). The tubes were allowed to draw laboratory air for two minutes $(50 \mathrm{cc} / \mathrm{min})$ to volatilize the analytes of interest before being connected to a Miller-Nelson atmosphere generator. Humidity-controlled air $(20 \%, 50 \%$, and $80 \%)$ was sampled for 240 minutes resulting in a total volume of $12 \mathrm{~L}$. The tubes were then refrigerated overnight. To determine whether breakthrough or migration had occurred during sampling, the sorbent from the individual sections of the tubes was removed and placed into individual $4 \mathrm{~mL}$ amber colored desorption vials required to prevent UV degradation of samples.

2.3.6. Capacity Studies. The initial collection capacity study was conducted to evaluate the effects of relative humidity on recovery. The custom-made $o$-PDA-treated silica gel tubes were placed on the air sampling manifold and the flow rate of the manifold was adjusted to $50 \mathrm{cc} / \mathrm{min}$. Each tube was then spiked with a mixture containing diacetyl, 2,3-pentanedione, and 2,3-hexanedione at concentrations of $1 \mu \mathrm{g}$ and $500 \mu \mathrm{g}$. Spikes were made on the glass wool preceding the sorbent and humid air was pulled through the tubes.

The tubes were allowed to draw laboratory air for two minutes to volatilize the analytes before being connected to an atmosphere generator to produce the humidity-controlled air $(20 \%$ and $80 \%)$. Humidity-controlled air was sampled for total volumes ranging from $3 \mathrm{~L}$ to $24 \mathrm{~L}$ (60 to 480 minutes). Sample preparation and analyses were conducted under the parameters previously described.

In an effort to evaluate the effect of an increased sampling rate $(200 \mathrm{cc} / \mathrm{min})$ and maximize sampling volumes collected, a more in-depth capacity study was conducted. In this study, 2,3-heptanedione was added as an analyte due to its continued presence as a minor component in alpha-dicarbonyl based flavoring compounds. The custommade $o$-PDA-treated silica gel tubes were placed on the air sampling manifold and the flow rate of the manifold was adjusted to $200 \mathrm{cc} / \mathrm{min}$. Each tube was then spiked on glass wool at the front section with a solution of diacetyl, 2,3pentanedione, 2,3-hexanedione, and 2,3-heptanedione at 2 levels $(N=3): 0.5 \mu \mathrm{g}$ and $100 \mu \mathrm{g}$. The tubes were allowed to draw laboratory air for two minutes to volatilize the analytes before being connected to an atmosphere generator to produce the humidity-controlled air $(20 \%, 50 \%$, and $80 \%)$.

Humidity-controlled air was sampled for total volumes ranging from $96 \mathrm{~L}$ to $288 \mathrm{~L}$ ( 480 to 1440 minutes). Tubes were collected from each volume sampled and placed in refrigerated storage overnight. Sample preparation and analyses were conducted under the parameters previously described.

2.3.7. Storage Stability Studies. To evaluate sample stability [13], custom-made $o$-PDA-treated silica gel tubes were spiked with $0.6 \mu \mathrm{g}$ each of diacetyl, 2,3-pentanedione, 2,3hexanedione, and 2,3-heptanedione as shown in Table 2. Six sorbent tubes were analyzed after 1, 7,14, and 30 days. Separate sets of samples were analyzed after storage under ambient and refrigerated storage conditions. Sample preparation and analyses were conducted under the parameters previously described. 
TABLE 2: Standard stock spiking solution preparation and spiking volumes for stability studies.

\begin{tabular}{lccccc}
\hline Analyte & $\begin{array}{c}\text { Amount neat analyte } \\
\text { spike }(\mu \mathrm{L})\end{array}$ & Final volume $(\mathrm{mL})$ & $\begin{array}{c}\text { Final concentration } \\
(\mu \mathrm{g} / \mathrm{mL})\end{array}$ & Volume spiked $(\mu \mathrm{L})$ & Amount spiked $(\mu \mathrm{g})$ \\
\hline Diacetyl & 1 & 10 & 98.5 & 6 & 0.591 \\
2,3-Pentanedione & 1 & 10 & 95.9 & 6 & 0.575 \\
2,3-Hexanedione (90\%) & 1 & 10 & 84.0 & 6 & 0.504 \\
2,3-Heptanedione & 1 & 10 & 92.0 & 6 & 0.552 \\
\hline
\end{tabular}

TABLE 3: Limit of detection (LOD) and Limit of Quantitation (LOQ) for alpha-dicarbonyl compounds.

\begin{tabular}{lcc}
\hline Analyte & $\mathrm{LOD}^{14}$ & $\mathrm{LOQ}^{14}$ \\
\hline Diacetyl & $7 \mathrm{ng} / \mathrm{mL}$ & $23 \mathrm{ng} / \mathrm{mL}$ \\
2,3-Pentanedione & $17 \mathrm{ng} / \mathrm{mL}$ & $58 \mathrm{ng} / \mathrm{mL}$ \\
$2,3-$ Hexanedione & $5 \mathrm{ng} / \mathrm{mL}$ & $15 \mathrm{ng} / \mathrm{mL}$ \\
\hline
\end{tabular}

TABLE 4: Diacetyl recovery after extraction of spiked sorbent with $o$-PDA solution.

\begin{tabular}{lcc}
\hline Spike level $(\mu \mathrm{g})$ & Average recovery $(\%)$ & RSD \\
\hline 0.096 & 56.3 & 0.094 \\
9.55 & 96.4 & 0.019 \\
95.5 & 93.0 & 0.032 \\
239.0 & 80.1 & 0.017 \\
478.0 & 104.3 & 0.033 \\
\hline
\end{tabular}

\section{Results}

3.1. LOD/LOQ Determination. As previously described, eight standards (in duplicate) were analyzed using GC-NPD: diacetyl (2.65 to $662.5 \mathrm{ng} / \mathrm{mL}$ ), 2,3-pentanedione (10 to $100.7 \mathrm{ng} / \mathrm{mL}$ ), and 2,3-hexanedione (5 to $201.6 \mathrm{ng} / \mathrm{mL}$ ). The instrumental LOD and LOQ were determined using calibration curves (diacetyl - slope $=904.66$, intercept $=15.74$, and $R^{2}=0.9216 ; 2,3$-pentanedione - slope $=533.19$, intercept $=$ 29.8 , and $R^{2}=0.7918 ; 2,3$-hexanedione - slope $=426.30$, intercept $=2.5$, and $R^{2}=0.9796$; and 2,3-heptanedione slope $=113.28$, intercept $=28.95$, and $R^{2}=0.9716$ ). Results are listed in Table 3.

3.2. Recovery Study (Untreated Silica Gel Sorbent Tubes). On the basis of the initial recovery results achieved when diacetyl was spiked directly on untreated silica gel tubes and desorbed in a solution of $1 \mathrm{mg} / \mathrm{mL}$ of $o$-PDA, a full scale recovery study was evaluated. Desorption efficiency recoveries for 2,3-dimethylquinoxaline (diacetyl derivative) ranged from $56.3 \%(0.0955 \mu \mathrm{g})$ to $104.3 \%(478 \mu \mathrm{g})$ with an average Relative Standard Deviation (RSD) of 0.039 are listed in Table 4.

3.3. Recovery Study (o-PDA-Treated Silica Gel Sorbent Tubes). The next phase in the method development process for the derivatization of diacetyl, 2,3-pentanedione, and 2,3hexanedione was to determine the feasibility of collecting and derivatizing the analytes "on-tube" using $o$-PDA-coated silica gel sorbent tubes. The initial glass wool plugs were
TABLE 5: Recovery results for the on-tube derivatization of diacetyl, 2,3-pentanedione, and 2,3-hexanedione.

\begin{tabular}{lccc}
\hline Analyte & Spike level $(\mu \mathrm{g})$ & $\begin{array}{c}\text { Average recovery } \\
(\%)\end{array}$ & RSD \\
\hline \multirow{3}{*}{ Diacetyl } & 0.096 & 87.0 & 0.106 \\
& 9.55 & 99.6 & 0.037 \\
& 95.5 & 91.4 & 0.099 \\
& 239.0 & 104.0 & 0.080 \\
& 478.0 & 103.0 & 0.077 \\
\hline \multirow{3}{*}{ 2,3-Pentanedione } & $0.96^{*}$ & $-^{*}$ & $-^{*}$ \\
& 9.59 & 106.5 & 0.045 \\
& 95.9 & 96.1 & 0.043 \\
& 240.0 & 92.3 & 0.064 \\
2,3-Hexanedione & 480.0 & 87.3 & 0.041 \\
& 0.117 & 69.7 & 0.212 \\
& 11.7 & 73.5 & 0.032 \\
& 292.0 & 69.6 & 0.032 \\
& 584.0 & 66.8 & 0.040 \\
\hline
\end{tabular}

${ }^{*}$ Data unavailable due to sample loss during analytical preparation.

spiked with diacetyl. Ambient air, generated by a MillerNelson atmospheric generator, was drawn through the tubes at $0.05 \mathrm{~L} / \mathrm{min}$. Desorption efficiencies for the analytes' derivatization with $o$-PDA are depicted in Table 5.

3.4. Low-Level Recovery Studies. As noted earlier in the Methods, after the successful recovery study at levels above $1 \mu \mathrm{g}$, a low-level recovery study was initiated. The recoveries for diacetyl ranged from $87.2 \%$ to $100.7 \%$ with an average RSD of 0.073 ; for 2,3-pentanedione ranged from $94.8 \%$ to $120.1 \%$ with an average RSD of 0.068 ; for 2,3-hexanedione ranged from $105.1 \%$ to $117.3 \%$ with an average RSD of 0.058 ; and for 2,3 -heptanedione ranged from $83.4 \%$ to $90.6 \%$ with an average RSD of 0.071 . Results are listed in Table 6.

3.5. Studies of the Effect of Humidity on Recovery. Due to the negative effects that humidity has on diacetyl recovery discovered during some of the more recent field sampling surveys conducted at food and flavoring sites using NIOSH method 2557 [5], the next progression in our method development effort was to evaluate the effect on sample collection of various levels of humidity when using the $o$-PDAcoated silica gel sorbent tubes. Spiking levels ranged from 
TABLE 6: Low level recovery study for diacetyl, 2,3-pentanedione, 2,3-hexanedione, and 2,3-heptanedione.

\begin{tabular}{lccc}
\hline Analyte & Spike level $(\mu \mathrm{g})$ & Recovery $(\%)$ & RSD \\
\hline \multirow{3}{*}{ Diacetyl } & 0.099 & 87.2 & 0.139 \\
& 0.591 & 96.7 & 0.046 \\
& 0.985 & 100.7 & 0.036 \\
\hline \multirow{3}{*}{ 2,3-Pentanedione } & 0.096 & 120.1 & 0.112 \\
& 0.575 & 94.8 & 0.051 \\
& 0.959 & 108.8 & 0.038 \\
\hline \multirow{3}{*}{ 2,3-Hexanedione } & 0.084 & 105.1 & 0.043 \\
& 0.504 & 117.3 & 0.056 \\
& 0.840 & 105.1 & 0.043 \\
\hline \multirow{3}{*}{ 2,3-Heptanedione } & 0.092 & 83.4 & 0.072 \\
& 0.552 & 90.5 & 0.071 \\
& 0.920 & 90.6 & 0.065 \\
\hline
\end{tabular}

approximately $0.1 \mu \mathrm{g}$ to $500 \mu \mathrm{g}$. After sample collection for a period of 240 minutes and a total volume of $12 \mathrm{~L}$, recoveries were determined for each derivatized analyte collected at relative humidities of $20 \%, 50 \%$, and $80 \%$ (actual measured humidity). The results are listed in Table 7.

3.6. Capacity Studies. In the initial collection capacity study, diacetyl, 2,3-pentanedione, and 2,3-hexanedione were sampled on the $o$-PDA-coated silica gel tubes at two levels $(1 \mu \mathrm{g}$ and $500 \mu \mathrm{g}$ ) for total air collection capacities ranging from $3 \mathrm{~L}(60 \mathrm{~min})$ to $24 \mathrm{~L}(480 \mathrm{~min})$. Sampling was conducted at relative humidities of $20 \%$ and $80 \%$ and the results are depicted in Tables 8 and 9.

In an effort to evaluate the effect of an increased sampling rate $(200 \mathrm{cc} / \mathrm{min})$ and maximize sampling volumes collected, a more in-depth capacity study was conducted. In this study, 2,3-heptanedione was added as an analyte due to its continued presence as a minor component (contaminant) in alpha-dicarbonyl based flavoring compounds. A more detailed depiction of the recovery data for each analyte is presented in Table 10 (20\% RH), Table 11 (50\% RH), and Table 12 (80\% RH). Mean recovery (\%) was calculated based on the average recovery of 3 samples evaluated at each volume sampled.

3.7. Storage Stability Recoveries. Evaluation of the ambient and refrigerated storage stability recovery results for diacetyl, 2,3-pentanedione, 2,3-hexanedione, and 2,3-heptanedione indicates that the derivatized analytes were stable for up to 30 days at the $0.6 \mu \mathrm{g}$ spiking levels. The average storage stability results evaluated for each analyte at 1, 7, 14, and 30 days are reported in Table 13.

\section{Discussion and Conclusions}

A method for alpha-dicarbonyl flavoring compounds has been developed using derivatization with $o$-PDA. This method has several advantages when compared to other
TABLE 7: Effects of varying humidity levels on analyte recovery.

\begin{tabular}{|c|c|c|c|c|}
\hline Analyte & Level $(\mu \mathrm{g})$ & $\begin{array}{c}\text { Relative } \\
\text { humidity } \\
(\%)\end{array}$ & $\begin{array}{l}\text { Mean } \\
\text { recovery }\end{array}$ & RSD \\
\hline \multirow{21}{*}{ Diacetyl } & 0.118 & 16.7 & 86.4 & 0.130 \\
\hline & 0.118 & 58.0 & 101.0 & 0.146 \\
\hline & 0.118 & 80.1 & 72.5 & 0.123 \\
\hline & 0.640 & 22.6 & 97.7 & 0.040 \\
\hline & 0.640 & 58.0 & 111.0 & 0.098 \\
\hline & 0.640 & 80.4 & 79.3 & 0.076 \\
\hline & 0.938 & 20.7 & 92.4 & 0.032 \\
\hline & 0.938 & 58.0 & 115.0 & 0.121 \\
\hline & 0.938 & 78.5 & 97.8 & 0.110 \\
\hline & 9.85 & 17.9 & 107.6 & 0.035 \\
\hline & 9.85 & 52.0 & 100.1 & 0.076 \\
\hline & 9.85 & 79.6 & 99.6 & 0.028 \\
\hline & 98.5 & 17.9 & 111.0 & 0.019 \\
\hline & 98.5 & 52.0 & 100.1 & 0.076 \\
\hline & 98.5 & 49.6 & 99.6 & 0.028 \\
\hline & 246.25 & 17.9 & 105.0 & 0.074 \\
\hline & 246.25 & 51.1 & 101.2 & 0.087 \\
\hline & 246.25 & 80.6 & 88.9 & 0.042 \\
\hline & 492.5 & 17.9 & 102.7 & 0.049 \\
\hline & 492.5 & 50.0 & 108.9 & 0.037 \\
\hline & 492.5 & 81.0 & 99.5 & 0.022 \\
\hline \multirow{21}{*}{$\begin{array}{l}2,3- \\
\text { Pentanedione }\end{array}$} & 0.115 & 16.7 & 63.9 & 0.133 \\
\hline & 0.115 & 58.0 & 98.7 & 0.112 \\
\hline & 0.115 & 80.1 & 73.9 & 0.123 \\
\hline & 0.622 & 22.6 & 88.4 & 0.031 \\
\hline & 0.622 & 58.0 & 85.6 & 0.080 \\
\hline & 0.622 & 80.4 & 76.7 & 0.074 \\
\hline & 0.909 & 20.7 & 84.9 & 0.026 \\
\hline & 0.909 & 58.0 & 102.0 & 0.142 \\
\hline & 0.909 & 78.5 & 98.8 & 0.137 \\
\hline & 9.59 & 21.1 & 94.8 & 0.072 \\
\hline & 9.59 & 51.3 & 85.8 & 0.071 \\
\hline & 9.59 & 80.8 & 64.1 & 0.107 \\
\hline & 95.9 & 21.1 & 102.7 & 0.085 \\
\hline & 95.9 & 51.4 & 100.0 & 0.102 \\
\hline & 95.9 & 79.7 & 46.2 & 0.060 \\
\hline & 239.75 & 21.1 & 104.9 & 0.051 \\
\hline & 239.75 & 50.7 & 99.1 & 0.119 \\
\hline & 239.75 & 80.6 & 73.9 & 0.110 \\
\hline & 479.5 & 21.1 & 90.2 & 0.084 \\
\hline & 479.5 & 50.4 & 87.1 & 0.125 \\
\hline & 479.5 & 79.9 & 80.3 & 0.112 \\
\hline
\end{tabular}


TABLE 7: Continued.

\begin{tabular}{|c|c|c|c|c|}
\hline Analyte & Level $(\mu \mathrm{g})$ & $\begin{array}{c}\text { Relative } \\
\text { humidity } \\
(\%)\end{array}$ & $\begin{array}{c}\text { Mean } \\
\text { recovery }\end{array}$ & RSD \\
\hline \multirow{21}{*}{$\begin{array}{l}2,3- \\
\text { Hexanedione }\end{array}$} & 0.101 & 16.7 & 120.0 & 0.195 \\
\hline & 0.101 & 58.0 & 114.3 & 0.085 \\
\hline & 0.101 & 80.1 & 49.9 & 0.062 \\
\hline & 0.546 & 22.6 & 108.0 & 0.038 \\
\hline & 0.546 & 58.0 & 109.0 & 0.060 \\
\hline & 0.546 & 80.4 & 82.4 & 0.064 \\
\hline & 0.799 & 20.7 & 103.8 & 0.034 \\
\hline & 0.799 & 58.0 & 124.0 & 0.206 \\
\hline & 0.799 & 78.5 & 90.8 & 0.107 \\
\hline & 11.68 & 21.1 & 67.8 & 0.055 \\
\hline & 11.68 & 51.3 & 60.4 & 0.056 \\
\hline & 11.68 & 80.8 & 45.4 & 0.078 \\
\hline & 116.75 & 21.1 & 66.4 & 0.051 \\
\hline & 116.75 & 51.4 & 68.6 & 0.060 \\
\hline & 116.75 & 79.7 & 31.1 & 0.039 \\
\hline & 291.88 & 21.1 & 66.8 & 0.028 \\
\hline & 291.88 & 50.7 & 72.1 & 0.107 \\
\hline & 291.88 & 80.6 & 40.4 & 0.080 \\
\hline & 583.75 & 21.1 & 59.6 & 0.057 \\
\hline & 583.75 & 50.4 & 52.5 & 0.079 \\
\hline & 583.75 & 79.9 & 53.7 & 0.088 \\
\hline \multirow{15}{*}{$\begin{array}{l}\text { 2,3- } \\
\text { Heptanedione }\end{array}$} & 0.110 & 16.7 & 90.3 & 0.180 \\
\hline & 0.110 & 58.0 & 69.8 & 0.142 \\
\hline & 0.110 & 80.1 & 75.9 & 0.062 \\
\hline & 0.598 & 22.6 & 81.0 & 0.034 \\
\hline & 0.598 & 58.0 & 88.7 & 0.066 \\
\hline & 0.598 & 80.4 & 78.4 & 0.061 \\
\hline & 0.874 & 20.7 & 75.3 & 0.067 \\
\hline & 0.874 & 58.0 & 108.0 & 0.195 \\
\hline & 0.874 & 78.5 & 107.0 & 0.111 \\
\hline & 9.20 & 21.5 & 84.9 & 0.099 \\
\hline & 9.20 & 47.3 & 84.5 & 0.053 \\
\hline & 9.20 & 80.3 & 81.9 & 0.077 \\
\hline & 92.0 & 21.5 & 103.1 & 0.044 \\
\hline & 92.0 & 52.7 & 92.3 & 0.030 \\
\hline & 92.0 & 83.8 & 90.2 & 0.031 \\
\hline
\end{tabular}

methods $[3,6,7]$ for alpha-dicarbonyl flavoring compounds, such as improved sensitivity (instrumental LODs of 5$17 \mathrm{ng} / \mathrm{sample}$ ), use of a single sampling tube amenable to ontube derivatization of the analytes of interest, longer sampling times, variable sampling rates, and greater sampling capacity (up to $288 \mathrm{~L}$ with low-to-moderate humidity). Chromatographic separation of the alpha-dicarbonyl derivatives was
TABLE 8: Determination of air sampling capacity of $o$-PDA-treated silica gel tubes at $20 \%$ relative humidity at 2 concentration levels.

\begin{tabular}{|c|c|c|c|c|}
\hline Analyte & Level $(\mu \mathrm{g})$ & $\begin{array}{c}\text { Sampling } \\
\text { volume }(\mathrm{L})\end{array}$ & $\begin{array}{c}\text { Mean } \\
\text { recovery }(\%)\end{array}$ & RSD \\
\hline \multirow{10}{*}{ Diacetyl } & 0.985 & 3 & 85.5 & 0.016 \\
\hline & 0.985 & 6 & 99.9 & 0.059 \\
\hline & 0.985 & 12 & 91.1 & 0.058 \\
\hline & 0.985 & 18 & 84.0 & 0.020 \\
\hline & 0.985 & 24 & 102.9 & 0.131 \\
\hline & 490.0 & 3 & 91.1 & 0.038 \\
\hline & 490.0 & 6 & 104.6 & 0.052 \\
\hline & 490.0 & 12 & 99.0 & 0.014 \\
\hline & 490.0 & 18 & 91.8 & 0.019 \\
\hline & 490.0 & 24 & 100.4 & 0.058 \\
\hline \multirow{10}{*}{$\begin{array}{l}2,3- \\
\text { Pentanedione }\end{array}$} & 0.959 & 3 & 105.2 & 0.056 \\
\hline & 0.959 & 6 & 103.3 & 0.085 \\
\hline & 0.959 & 12 & 105.8 & 0.053 \\
\hline & 0.959 & 18 & 85.8 & 0.016 \\
\hline & 0.959 & 24 & 106.7 & 0.098 \\
\hline & 480.0 & 3 & 96.3 & 0.038 \\
\hline & 480.0 & 6 & 97.0 & 0.045 \\
\hline & 480.0 & 12 & 110.6 & 0.028 \\
\hline & 480.0 & 18 & 116.6 & 0.077 \\
\hline & 480.0 & 24 & 102.3 & 0.039 \\
\hline \multirow{10}{*}{$\begin{array}{l}2,3- \\
\text { Hexanedione }\end{array}$} & 0.841 & 3 & 72.7 & 0.087 \\
\hline & 0.841 & 6 & 91.8 & 0.077 \\
\hline & 0.841 & 12 & 110.8 & 0.109 \\
\hline & 0.841 & 18 & 85.0 & 0.016 \\
\hline & 0.841 & 24 & 92.6 & 0.114 \\
\hline & 420.0 & 3 & 89.6 & 0.036 \\
\hline & 420.0 & 6 & 86.4 & 0.089 \\
\hline & 420.0 & 12 & 90.8 & 0.059 \\
\hline & 420.0 & 18 & 110.0 & 0.032 \\
\hline & 420.0 & 24 & 108.5 & 0.103 \\
\hline
\end{tabular}

good and the overall recovery of the analytes of interest down to the $0.1 \mu \mathrm{g}$ level was acceptable.

Diacetyl recoveries on untreated silica gel tubes following by desorption in ethanol containing the $o$-PDA derivatizing agent were acceptable at all spiking levels except the lowest (0.096 ng).

Recoveries of diacetyl and 2,3-pentanedione from the silica gel tubes coated with $o$-phenylenediamine were very good while the recoveries for 2,3-hexanedione were approximately $20 \%$ lower. Lower recoveries of 2,3-hexanedione and 2,3-heptanedione may be the result of the increasing hydrocarbon nature of these compounds and/or the fact that they possibly require an increased derivatization period. In addition, when larger amounts of the analyte were evaluated, some lower recoveries were found. This may be the result of incomplete derivatization and the need for a greater concentration of the derivatizing reagent on the sorbent 
TABLE 9: Determination of air sampling capacity of $o$-PDA-treated silica gel tubes at $80 \%$ relative humidity at 2 concentration levels.

\begin{tabular}{|c|c|c|c|c|}
\hline Analyte & Level $(\mu \mathrm{g})$ & $\begin{array}{l}\text { Sampling } \\
\text { volume }(\mathrm{L})\end{array}$ & $\begin{array}{c}\text { Mean } \\
\text { recovery (\%) }\end{array}$ & RSD \\
\hline \multirow{10}{*}{ Diacetyl } & 0.985 & 3 & 78.1 & 0.016 \\
\hline & 0.985 & 6 & 82.1 & 0.058 \\
\hline & 0.985 & 12 & 95.2 & 0.097 \\
\hline & 0.985 & 18 & 82.4 & 0.034 \\
\hline & 0.985 & 24 & 103.6 & 0.028 \\
\hline & 490.0 & 3 & 97.4 & 0.047 \\
\hline & 490.0 & 6 & 88.5 & 0.074 \\
\hline & 490.0 & 12 & 112.7 & 0.034 \\
\hline & 490.0 & 18 & 100.6 & 0.018 \\
\hline & 490.0 & 24 & 93.7 & 0.065 \\
\hline \multirow{10}{*}{$\begin{array}{l}2,3- \\
\text { Pentanedione }\end{array}$} & 0.959 & 3 & 100.1 & 0.030 \\
\hline & 0.959 & 6 & 76.1 & 0.051 \\
\hline & 0.959 & 12 & 101.2 & 0.068 \\
\hline & 0.959 & 18 & 97.8 & 0.048 \\
\hline & 0.959 & 24 & 81.1 & 0.139 \\
\hline & 480.0 & 3 & 97.8 & 0.055 \\
\hline & 480.0 & 6 & 92.3 & 0.085 \\
\hline & 480.0 & 12 & 99.2 & 0.058 \\
\hline & 480.0 & 18 & 87.1 & 0.010 \\
\hline & 480.0 & 24 & 85.2 & 0.071 \\
\hline \multirow{10}{*}{$\begin{array}{l}2,3- \\
\text { Hexanedione }\end{array}$} & 0.841 & 3 & 100.7 & 0.018 \\
\hline & 0.841 & 6 & 69.5 & 0.077 \\
\hline & 0.841 & 12 & 98.3 & 0.056 \\
\hline & 0.841 & 18 & 101.4 & 0.027 \\
\hline & 0.841 & 24 & 80.4 & 0.141 \\
\hline & 420.0 & 3 & 95.5 & 0.049 \\
\hline & 420.0 & 6 & 92.5 & 0.086 \\
\hline & 420.0 & 12 & 87.7 & 0.053 \\
\hline & 420.0 & 18 & 94.7 & 0.048 \\
\hline & 420.0 & 24 & 87.6 & 0.173 \\
\hline
\end{tabular}

media at these higher levels and/or the fact that the higher concentrations evaluated may exceed the sampling capacity of the sorbent tubes. The recoveries for 2,3-hexanedione and 2,3-heptanedione are lower than what is normally considered acceptable [13]. While this method was developed for diacetyl and 2,3-pentanedione measurement to address humidity issues with existing methods, it can be used to determine the presence of larger chain alpha-dicarbonyl compounds that may be present as by-products.

Sample collection for diacetyl was unaffected by humidity ranging from $20 \%$ to $80 \%$. For the other flavoring agents tested, high humidity reduced the recovery. While additional research studies are ongoing, it can be reasonably concluded that this method has achieved significant advancements in the sampling and quantitation of alpha-dicarbonyl flavoring compounds. Overall, recoveries were good for diacetyl when sampled in conditions of $80 \%$ humidity.
TABLE 10: Determination of extended air sampling capacity of $o$ PDA-treated silica gel tubes at $20 \%$ relative humidity at 2 concentration levels using increased sampling rate $(200 \mathrm{cc} / \mathrm{min})$.

\begin{tabular}{|c|c|c|c|c|c|}
\hline Analyte & $\begin{array}{c}\text { Level } \\
(\mu \mathrm{g})\end{array}$ & $\begin{array}{l}\text { Sampling } \\
\text { volume } \\
(\mathrm{L})\end{array}$ & $\begin{array}{l}\text { Time } \\
(\min )\end{array}$ & $\begin{array}{c}\text { Mean } \\
\text { recovery } \\
(\%)\end{array}$ & RSD \\
\hline \multirow{8}{*}{ Diacetyl } & 0.493 & 96 & 480 & 99.8 & 0.035 \\
\hline & 0.493 & 144 & 720 & 110.0 & 0.040 \\
\hline & 0.493 & 216 & 960 & 104.0 & 0.054 \\
\hline & 0.493 & 288 & 1440 & 108.0 & 0.043 \\
\hline & 98.5 & 96 & 480 & 98.3 & 0.010 \\
\hline & 98.5 & 144 & 720 & 104.0 & 0.023 \\
\hline & 98.5 & 216 & 960 & 100.0 & 0.038 \\
\hline & 98.5 & 288 & 1440 & 95.5 & 0.073 \\
\hline \multirow{8}{*}{$\begin{array}{l}2,3- \\
\text { Pentanedione }\end{array}$} & 0.479 & 96 & 480 & 92.1 & 0.015 \\
\hline & 0.479 & 144 & 720 & 83.0 & 0.046 \\
\hline & 0.479 & 216 & 960 & 101.0 & 0.088 \\
\hline & 0.479 & 288 & 1440 & 109.0 & 0.181 \\
\hline & 95.7 & 96 & 480 & 97.8 & 0.038 \\
\hline & 95.7 & 144 & 720 & 99.4 & 0.021 \\
\hline & 95.7 & 216 & 960 & 103.0 & 0.156 \\
\hline & 95.7 & 288 & 1440 & 95.8 & 0.046 \\
\hline \multirow{8}{*}{$\begin{array}{l}2,3- \\
\text { Hexanedione }\end{array}$} & 0.420 & 96 & 480 & 97.1 & 0.021 \\
\hline & 0.420 & 144 & 720 & 102.0 & 0.036 \\
\hline & 0.420 & 216 & 960 & 109.0 & 0.016 \\
\hline & 0.420 & 288 & 1440 & 106.0 & 0.061 \\
\hline & 84.1 & 96 & 480 & 87.9 & 0.036 \\
\hline & 84.1 & 144 & 720 & 101.0 & 0.021 \\
\hline & 84.1 & 216 & 960 & 99.4 & 0.032 \\
\hline & 84.1 & 288 & 1440 & 84.6 & 0.047 \\
\hline \multirow{8}{*}{$\begin{array}{l}2,3- \\
\text { Heptanedione }\end{array}$} & 0.460 & 96 & 480 & 104.0 & 0.027 \\
\hline & 0.460 & 144 & 720 & 101.0 & 0.065 \\
\hline & 0.460 & 216 & 960 & 106.0 & 0.025 \\
\hline & 0.460 & 288 & 1440 & 108.0 & 0.052 \\
\hline & 92.0 & 96 & 480 & 93.9 & 0.082 \\
\hline & 92.0 & 144 & 720 & 98.6 & 0.029 \\
\hline & 92.0 & 216 & 960 & 104.0 & 0.024 \\
\hline & 92.0 & 288 & 1440 & 93.5 & 0.052 \\
\hline
\end{tabular}

The resulting recovery for 2,3-pentanedione at $95.9 \mu \mathrm{g}$ (46.2\%) is abnormally low when compared to all other results and is most likely an aberration when compared to the results listed in Table 9 for 2,3-pentanedione. Recoveries for both 2,3-hexanedione and 2,3-heptanedione were lower than expected for those samples collected at $80 \%$ humidity in the extended air sampling capacity studies. These analytes are present as by-products or contaminants with either diacetyl or 2,3-pentanedione. This method can be used to detect these contaminants where other methods cannot.

In laboratory capacity studies, where diacetyl, 2,3pentanedione, 2,3-hexanedione, and 2,3-heptanedione were collected at $20 \%$ relative humidity and with a sampling 
TABLE 11: Determination of extended air sampling capacity of $o$ PDA-treated silica gel tubes at $50 \%$ relative humidity at 2 concentration levels using increased sampling rate $(200 \mathrm{cc} / \mathrm{min})$.

\begin{tabular}{|c|c|c|c|c|c|}
\hline Analyte & $\begin{array}{c}\text { Level } \\
(\mu \mathrm{g})\end{array}$ & $\begin{array}{l}\text { Sampling } \\
\text { volume } \\
\text { (L) }\end{array}$ & $\begin{array}{l}\text { Time } \\
(\min )\end{array}$ & $\begin{array}{c}\text { Mean } \\
\text { recovery } \\
(\%)\end{array}$ & RSD \\
\hline \multirow{8}{*}{ Diacetyl } & 0.493 & 96 & 480 & 102.0 & 0.015 \\
\hline & 0.493 & 144 & 720 & 92.6 & 0.024 \\
\hline & 0.493 & 216 & 960 & 102.0 & 0.018 \\
\hline & 0.493 & 288 & 1440 & 87.0 & 0.026 \\
\hline & 98.5 & 96 & 480 & 98.8 & 0.027 \\
\hline & 98.5 & 144 & 720 & 106.0 & 0.016 \\
\hline & 98.5 & 216 & 960 & 101.8 & 0.020 \\
\hline & 98.5 & 288 & 1440 & 101.0 & 0.028 \\
\hline \multirow{8}{*}{$\begin{array}{l}2,3- \\
\text { Pentanedione }\end{array}$} & 0.479 & 96 & 480 & 92.7 & 0.010 \\
\hline & 0.479 & 144 & 720 & 77.8 & 0.054 \\
\hline & 0.479 & 216 & 960 & 98.5 & 0.158 \\
\hline & 0.479 & 288 & 1440 & 88.5 & 0.009 \\
\hline & 95.7 & 96 & 480 & 97.8 & 0.030 \\
\hline & 95.7 & 144 & 720 & 100.0 & 0.019 \\
\hline & 95.7 & 216 & 960 & 110.0 & 0.024 \\
\hline & 95.7 & 288 & 1440 & 97.8 & 0.026 \\
\hline \multirow{8}{*}{$\begin{array}{l}2,3- \\
\text { Hexanedione }\end{array}$} & 0.420 & 96 & 480 & 93.7 & 0.024 \\
\hline & 0.420 & 144 & 720 & 86.2 & 0.021 \\
\hline & 0.420 & 216 & 960 & 87.9 & 0.020 \\
\hline & 0.420 & 288 & 1440 & 71.4 & 0.018 \\
\hline & 84.1 & 96 & 480 & 88.0 & 0.023 \\
\hline & 84.1 & 144 & 720 & 102.0 & 0.023 \\
\hline & 84.1 & 216 & 960 & 103.0 & 0.026 \\
\hline & 84.1 & 288 & 1440 & 89.3 & 0.018 \\
\hline \multirow{8}{*}{$\begin{array}{l}2,3- \\
\text { Heptanedione }\end{array}$} & 0.460 & 96 & 480 & 103.0 & 0.019 \\
\hline & 0.460 & 144 & 720 & 89.3 & 0.026 \\
\hline & 0.460 & 216 & 960 & 97.8 & 0.031 \\
\hline & 0.460 & 288 & 1440 & 98.5 & 0.041 \\
\hline & 92.0 & 96 & 480 & 94.1 & 0.020 \\
\hline & 92.0 & 144 & 720 & 99.8 & 0.017 \\
\hline & 92.0 & 216 & 960 & 105.0 & 0.011 \\
\hline & 92.0 & 288 & 1440 & 98.6 & 0.019 \\
\hline
\end{tabular}

rate of $200 \mathrm{cc} / \mathrm{min}$, all three analytes exhibited acceptable recoveries $(>80 \%)$ with little variation in the mean recoveries when sampling for a total volume of $288 \mathrm{~L}$. At $50 \%$ relative humidity, diacetyl, 2,3-pentanedione, 2,3-hexanedione, and 2,3-heptanedione exhibited acceptable recoveries. Results for the samples collected for the $100 \mu \mathrm{g}$ level showed that 2,3pentanedione, 2,3-hexanedione, and 2,3-heptanedione had significant decreases in recovery at a collection volume of $216 \mathrm{~L}$. These results would seem to suggest that the maximum sampling volume for these analytes, when collected at a higher sampling rate $(200 \mathrm{cc} / \mathrm{min})$, would be between 144 and $216 \mathrm{~L}$.
TABLE 12: Determination of extended air sampling capacity of $o$ PDA-treated silica gel tubes at $80 \%$ relative humidity at 2 concentration levels using increased sampling rate $(200 \mathrm{cc} / \mathrm{min})$.

\begin{tabular}{|c|c|c|c|c|c|}
\hline Analyte & $\begin{array}{c}\text { Level } \\
(\mu \mathrm{g})\end{array}$ & $\begin{array}{c}\text { Sampling } \\
\text { volume } \\
\text { (L) }\end{array}$ & $\begin{array}{l}\text { Time } \\
(\mathrm{min})\end{array}$ & $\begin{array}{c}\text { Mean } \\
\text { recovery } \\
(\%)\end{array}$ & RSD \\
\hline \multirow{8}{*}{ Diacetyl } & 0.985 & 96 & 480 & 99.7 & 0.017 \\
\hline & 0.985 & 144 & 720 & 101.0 & 0.110 \\
\hline & 0.985 & 216 & 960 & 93.7 & 0.055 \\
\hline & 0.985 & 288 & 1440 & 84.1 & 0.114 \\
\hline & 98.5 & 96 & 480 & 90.5 & 0.072 \\
\hline & 98.5 & 144 & 720 & 98.8 & 0.036 \\
\hline & 98.5 & 216 & 960 & 93.5 & 0.057 \\
\hline & 98.5 & 288 & 1440 & 89.4 & 0.037 \\
\hline \multirow{8}{*}{$\begin{array}{l}2,3- \\
\text { Pentanedione }\end{array}$} & 0.957 & 96 & 480 & 72.9 & 0.081 \\
\hline & 0.957 & 144 & 720 & 112.0 & 0.055 \\
\hline & 0.957 & 216 & 960 & 105.0 & 0.114 \\
\hline & 0.957 & 288 & 1440 & 99.5 & 0.054 \\
\hline & 95.7 & 96 & 480 & 86.0 & 0.055 \\
\hline & 95.7 & 144 & 720 & 93.8 & 0.038 \\
\hline & 95.7 & 216 & 960 & 55.2 & 0.054 \\
\hline & 95.7 & 288 & 1440 & 48.8 & 0.035 \\
\hline \multirow{8}{*}{$\begin{array}{l}2,3- \\
\text { Hexanedione }\end{array}$} & 0.841 & 96 & 480 & 119.0 & 0.138 \\
\hline & 0.841 & 144 & 720 & 122.0 & 0.062 \\
\hline & 0.841 & 216 & 960 & 105.0 & 0.114 \\
\hline & 0.841 & 288 & 1440 & 44.8 & 0.031 \\
\hline & 84.1 & 96 & 480 & 86.2 & 0.060 \\
\hline & 84.1 & 144 & 720 & 93.5 & 0.039 \\
\hline & 84.1 & 216 & 960 & 60.4 & 0.053 \\
\hline & 84.1 & 288 & 1440 & 54.6 & 0.015 \\
\hline \multirow{8}{*}{$\begin{array}{l}2,3- \\
\text { Heptanedione }\end{array}$} & 0.920 & 96 & 480 & 52.0 & 0.021 \\
\hline & 0.920 & 144 & 720 & 104.0 & 0.089 \\
\hline & 0.920 & 216 & 960 & 76.0 & 0.154 \\
\hline & 0.920 & 288 & 1440 & 57.2 & 0.055 \\
\hline & 92.0 & 96 & 480 & 85.5 & 0.058 \\
\hline & 92.0 & 144 & 720 & 93.7 & 0.036 \\
\hline & 92.0 & 216 & 960 & 57.8 & 0.050 \\
\hline & 92.0 & 288 & 1440 & 53.7 & 0.018 \\
\hline
\end{tabular}

The average 30-day storage stability recovery results, almost quantitative in nature, are extremely good and acceptable for both the ambient and refrigerated samples of the derivatized diacetyl and 2,3-pentanedione, suggesting that temperature does not have either a positive or a negative effect on the derivatization and storage of the diacetyl samples. Since both the ambient and refrigerated samples had quantitative recoveries ( $>95 \%)$ and RSD values less than $1 \%$ [13], there is no difference between the two methods of storage.

Evaluation of both ambient and refrigerated storage stability recovery results for 2,3-hexanedione indicates that the derivatized analyte was stable for up to 30 days at the $0.6 \mu \mathrm{g}$ 
TABLE 13: Storage stability conducted under ambient and refrigerated storage conditions.

\begin{tabular}{lcccc}
\hline Day & $\begin{array}{c}\text { Avg. recovery } \\
\text { (ambient, \%) }\end{array}$ & RSD & $\begin{array}{c}\text { Avg. recovery } \\
\text { (refrigerated, \%) }\end{array}$ & RSD \\
\hline \multicolumn{5}{c}{ Diacetyl } \\
1 & 96.1 & 0.068 & 96.7 & 0.046 \\
7 & 93.4 & 0.048 & 89.8 & 0.039 \\
14 & 98.6 & 0.017 & 106.0 & 0.065 \\
30 & 103.0 & 0.039 & 98.6 & 0.047 \\
\hline \multicolumn{5}{c}{2,3 -Pentanedione } \\
1 & 98.0 & 0.049 & 94.8 & 0.051 \\
7 & 102.0 & 0.045 & 100.0 & 0.025 \\
14 & 105.0 & 0.091 & 97.1 & 0.068 \\
30 & 108.0 & 0.065 & 96.8 & 0.073 \\
\hline \multicolumn{5}{c}{2,3 -Hexanedione } \\
1 & 109.8 & 0.115 & 117.0 & 0.056 \\
7 & 93.9 & 0.041 & 110.0 & 0.054 \\
14 & 87.0 & 0.068 & 99.0 & 0.040 \\
30 & 81.4 & 0.026 & 99.6 & 0.053 \\
\hline \multicolumn{5}{c}{$2,3-$ Heptanedione } \\
1 & 89.3 & 0.109 & 90.5 & 0.072 \\
7 & 90.8 & 0.055 & 84.9 & 0.059 \\
14 & 92.2 & 0.053 & 83.6 & 0.096 \\
30 & 92.2 & 0.021 & 97.0 & 0.082 \\
\hline
\end{tabular}

spiking levels. Analysis of the results indicates that there is improved storage stability (18\% increase in average recovery) when the samples are refrigerated. This is especially true for the samples analyzed after 14 and 30 days. Evaluation of both ambient and refrigerated storage stability recovery results for 2,3-heptanedione indicates that the derivatized analyte was stable for 30 days. Comparison of the averaged recoveries for both the ambient and refrigerated samples of the derivatized 2,3-heptanedione revealed no differences based on storage temperature. Storage stability studies indicated that the compounds of interest, especially diacetyl and 2,3-pentanedione, as their quinoxaline derivatives, are stable at both ambient and refrigerated temperatures for 30 days. Separation of other alpha-dicarbonyls such as 2,3-hexanedione and 2,3heptanedione can be achieved with this method and provide semiquantitative results. Overall, this method may be another useful tool for the evaluation and monitoring of workers exposed to airborne alpha-dicarbonyl food and flavoring compounds. Additional laboratory and field studies using the method are necessary to obtain full validation and publication in the NIOSH Manual of Analytical Methods (NMAM).

In summary, to date, all results suggest that this method provides the sensitivity needed for nanogram level sampling for alpha-dicarbonyl food and flavoring compounds (diacetyl and 2,3-pentanedione). Additionally, the method allows collection over a wide mass range and at relative humidities ranging from $20 \%$ to $80 \%$, with acceptable recoveries achieved up to sampling volumes of $144 \mathrm{~L}$ and $288 \mathrm{~L}$ for 2,3pentanedione and diacetyl, respectively. The sampling and analytical methodology has been unaffected by breakthrough when sampling at high flow rates $(200 \mathrm{cc} /$ minute) and high sample collection volumes (144 L), eliminating the need for a second sorbent tube in series with the backup section of the single sorbent tube collecting any sample that breaks through. Additionally, the on-tube derivatization eliminates humidityrelated breakthrough of the alpha-dicarbonyl flavoring compounds by forming the stable quinoxaline derivatives.

\section{Competing Interests}

The authors declare that they have no competing interests.

\section{Acknowledgments}

The authors would like to thank Dr. Gayle DeBord and T. J. Lentz for their assistance and advice during the preparation of this paper.

\section{References}

[1] NIOSH, "NIOSH health hazard evaluation report," NIOSH HETA 2000-0401-2991, US Department of Health and Human Services, Centers for Disease Control and Prevention, National Institute for Occupational Safety and Health, Cincinnati, Ohio, USA, 2006.

[2] K. Kreiss, A. Gomaa, G. Kullman, K. Fedan, E. J. Simoes, and P. L. Enright, "Clinical bronchiolitis obliterans in workers at a microwave-popcorn plant," The New England Journal of Medicine, vol. 347, no. 5, pp. 330-338, 2002.

[3] S. M. Pendergrass, "Method development for the determination of diacetyl and acetoin at a microwave popcorn plant," Environmental Science and Technology, vol. 38, no. 3, pp. 858-861, 2004.

[4] K. Ashley, L. McKernan-Taylor, E. Burroughs, J. Deddens, S. M. Pendergrass, and R. P. Streicher, "Analytical performance criteria," Journal of Occupational and Environmental Hygiene, vol. 5, no. 11, pp. D111-D116, 2008.

[5] J. Cox-Ganser, G. Ganser, R. Saito et al., "Correcting diacetyl concentrations from air samples collected with NIOSH method 2557," Journal of Occupational and Environmental Hygiene, vol. 8, no. 2, pp. 59-70, 2011.

[6] OSHA, OSHA Method PV2118: Acetoin and Diacetyl, U.S. Department of Labor, Occupational Safety and Health Administration, UT Technical Center, Salt Lake City, Utah, USA, 2003.

[7] OSHA, OSHA Method 1013: Acetoin and Diacetyl, U.S. Department of Labor, Occupational Safety and Health Administration, UT Technical Center, Salt Lake City, Utah, USA, 2008.

[8] NIOSH, Occupational Exposure to Diacetyl and 2,3Pentanedione, CDC/NIOSH Draft Criteria for a Recommended Standard, 2011.

[9] OSHA, "OSHA Method 1012: Acetoin and Diacetyl. U.S. Department of Labor, Occupational Safety and Health Administration," Tech. Rep., UT Technical Center, Salt Lake City, Utah, USA, 2008.

[10] K. Fujioka and T. Shibamoto, "Determination of toxic carbonyl compounds in cigarette smoke," Environmental Toxicology, vol. 21, no. 1, pp. 47-54, 2006. 
[11] A. Barros, J. A. Rodriques, P. J. Almeida, and M. T. Oliva Teles, "Determination of glyoxal, methylglyoxal, and diacetyl in selected beer and wine, by HPLC with UV spectrophotometric detection, after derivatization with o-phenylenediamine," Journal of Liquid Chromatography \& Related Technologies, vol. 22, no. 13, pp. 2061-2069, 1999.

[12] J. A. Rodrigues, A. A. Barros, and P. G. Rodrigues, "Differential pulse polarographic determination of $\alpha$-dicarbonyl compounds in foodstuffs after derivatization with $o$-phenylenediamine," Journal of Agricultural and Food Chemistry, vol. 47, no. 8, pp. 3219-3222, 1999.

[13] E. R. Kennedy, T. J. Fishback, R. Song, P. M. Eller, and S. A. Shulman, Guidelines for Air Sampling and Analytical Method Development and Evaluation, SOP 018, U.S. Department of Health and Human Services, Public Health Service, Centers for Disease Control and Prevention, National Institute for Occupational Safety and Health, 1995. 


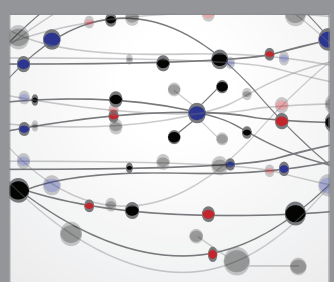

The Scientific World Journal
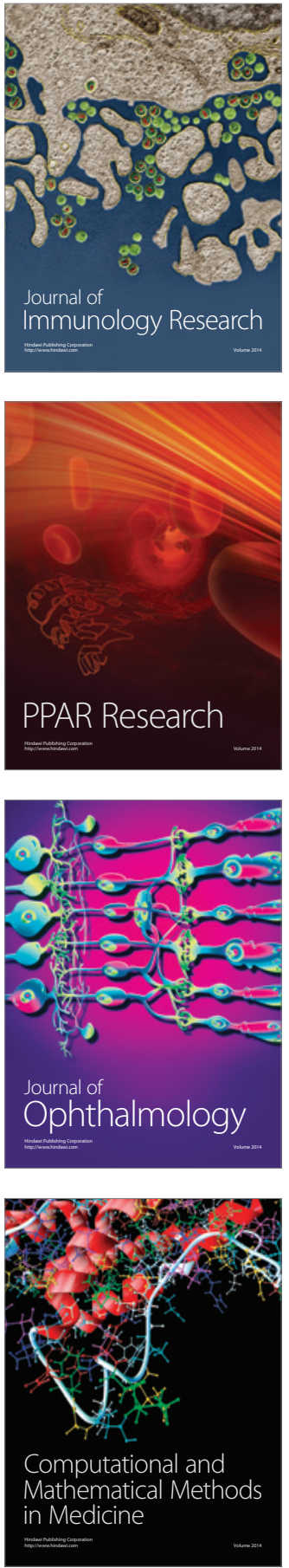

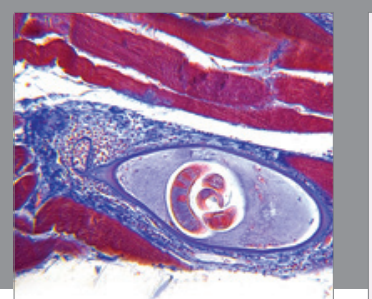

Gastroenterology Research and Practice

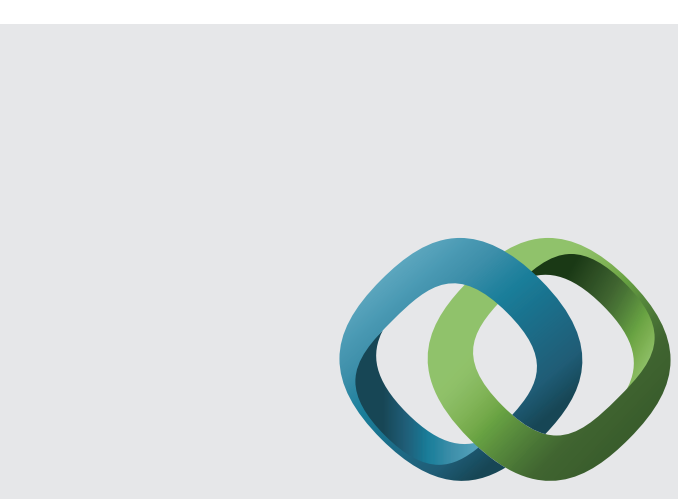

\section{Hindawi}

Submit your manuscripts at

http://www.hindawi.com
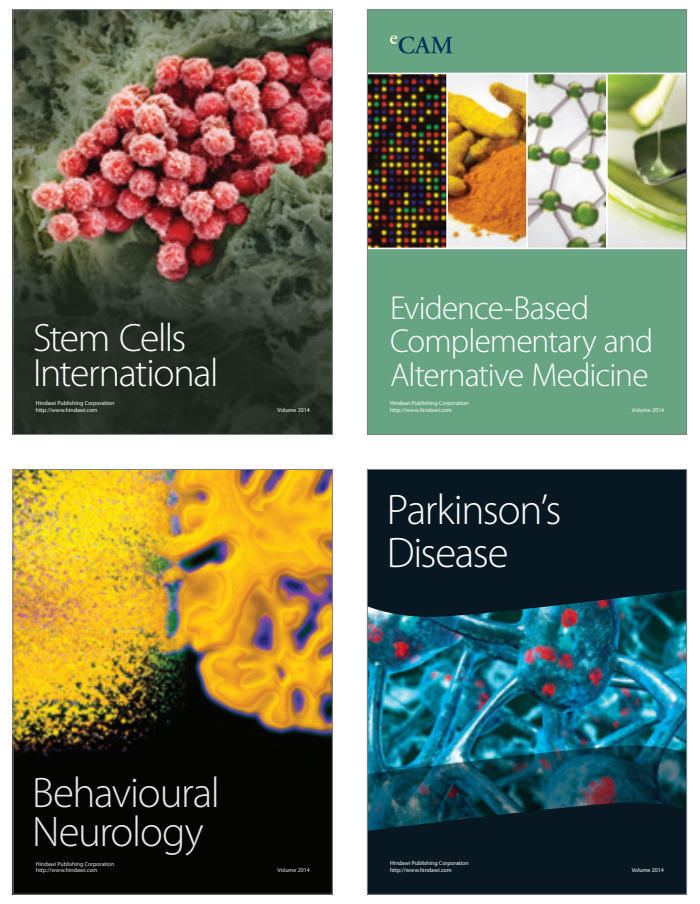
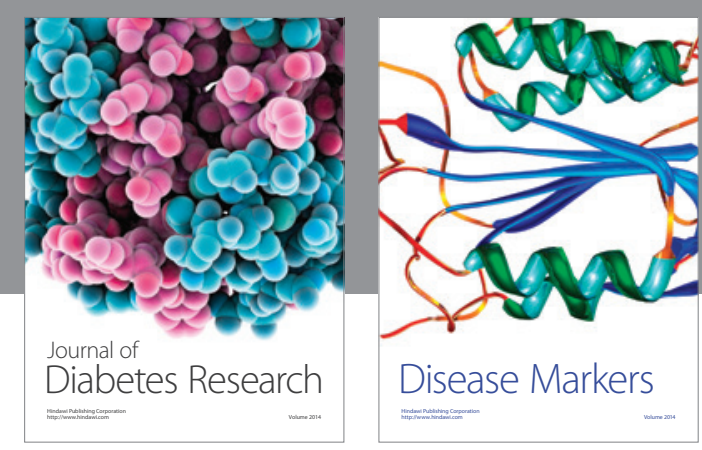

Disease Markers
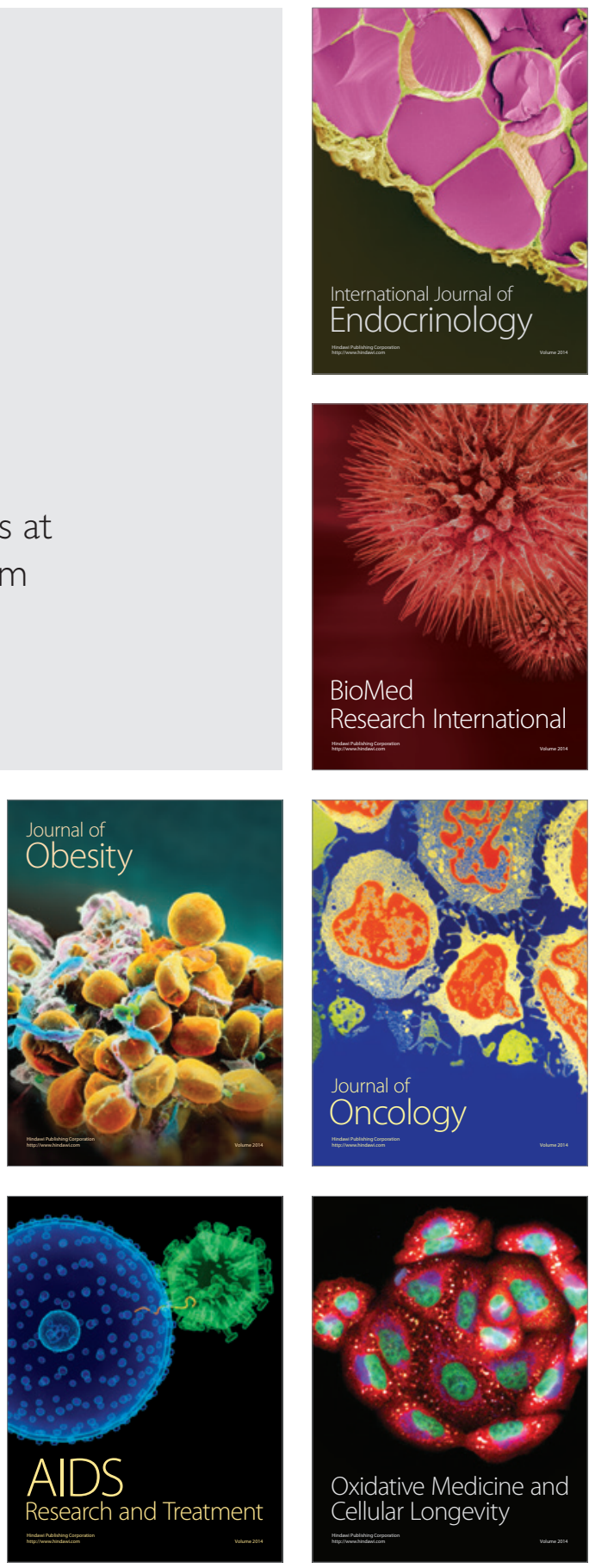